\title{
Investigación educativa: problemática y carácter multidisciplinario. Reflexiones teóricas y filosóficas
}

\author{
Ana María Valle Vázquez ${ }^{1}$ \\ Universidad Nacional Autónoma de México, México
}

Autor de correspondencia: 'anvallev@gmail.com

Recibido: 18 de julio de 2020 Revisado: 30 de julio de 2020 Aprobado: 21 de septiembre de 2020 Publicado: 25 de enero de 2021

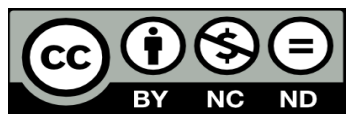

\section{Resumen}

El texto es el resultado de una reflexión cuyo principal objetivo es indagar en torno a la investigación educativa y su carácter multidisciplinario. En un primer momento, se asume que, por un lado, la problemática de la investigación educativa está relacionada con el sentido conceptual y teóricometodológico que nace del pensamiento y de la crítica a las preguntas por la educación. Por otra parte, el prefijo multino solo hace referencia a la abundancia y a la diversidad; también alude a las intersecciones, desvíos, irrupciones y articulaciones de las disciplinas. En un segundo momento, se revisa la investigación educativa desde la disciplinarización del saber como un combate entre saberes muy propio de la modernidad. El planteamiento teórico metodológico que se sigue es la hermenéutica. Se considera que toda interpretación tiene que protegerse contra la arbitrariedad de las ocurrencias y, más bien, debe orientar su mirada a la "cosa misma". En este caso, esta mirada interpretativa se dirige a la comprensión de la problemática de la investigación educativa y su carácter multidisciplinario.

Palabras clave: investigación educativa, metodología de la investigación, multidisciplinariedad, saber pedagógico, filosofía de la educación 


\title{
Educational research: issues and multidisciplinary nature. Theoretical and philosophical reflections
}

\begin{abstract}
The text is the result of a reflection that aims to investigate educational research and its multidisciplinary nature. At first, it is assumed that, on the one hand, the problem of educational research is related to the conceptual and theoretical-methodological meaning that arises from thinking and from the critique to the questions about education. On the other hand, the prefix multi- not only refers to abundance and diversity; it also alludes to the intersections, detours, irruptions, and linkages of the disciplines. Secondly, educational research is reviewed from the point of view of the disciplining of knowledge as a struggle between different types of knowledge that is very characteristic of the modern world. The theoretical-methodological approach adopted is hermeneutics. It is considered that any interpretation must be protected against the arbitrariness of the occurrences and, rather, must look at the "thing itself". In this case, this interpretative perspective is aimed at understanding the issues of educational research and its multidisciplinary nature.
\end{abstract}

Keywords: educational research, research methodology, multidisciplinary, pedagogical knowledge, educational philosophy

\section{Investigação educacional: problemas e carácter multidisciplinar. Reflexões teóricas e filosóficas}

\section{Resumo}

O texto é resultado de uma reflexão cujo principal objetivo é indagar sobre a pesquisa educacional e seu caráter multidisciplinar. Num primeiro momento, pressupõe-se que, por um lado, o problema da pesquisa educacional está relacionado ao sentido conceitual e teórico-metodológico que surge a partir do pensamento e da crítica às questões sobre a educação. Por outro lado, o prefixo multi- não se refere apenas à abundância e diversidade; também alude às intersecções, desvios, irrupções e articulações das disciplinas. Num segundo momento, a pesquisa educacional é revisada a partir da disciplinarização do conhecimento como um combate entre saberes bem típico da modernidade. A abordagem teórico-metodológica seguida é a hermenêutica. Considera-se que qualquer interpretação deve ser protegida contra a arbitrariedade das ocorrências e, em vez disso, deve dirigir seu olhar para a "coisa em si". Nesse caso, este olhar interpretativo é direcionado para a compreensão do problema da investigação educativa e seu carácter multidisciplinar.

Palavras-chave: investigação pedagógica, metodologia de investigação, multidisciplinaridade, saber pedagógico, filosofia da educação 
El conocimiento que procede de la investigación [educativa en particular] no es ciertamente la solución a los graves problemas del presente; pero cuando va unido a un compromiso vital y existencial, es una energía que se difunde y que puede detonar procesos positivos en todos aquellos actores de los que depende el destino de la educación

El texto está dividido en dos apartados: en el primero se reflexiona sobre lo "multi" de la investigación educativa, entre humanidades y ciencias sociales. Se identifica el carácter multidimensional y multirreferencial de la educación desde la orientación de los interrogantes, las ciencias sociales y las humanidades; en un segundo momento, se analiza, desde Foucault, la disciplinarización del saber —educativo y pedagógico一, así como su institucionalización.

Como ejercicio hermenéutico, hablar de la problemática —en singular- puede remitirnos a una reflexión de carácter conceptual y teórico; mientras que referirnos a las problemáticas - en plural- puede llevarnos a un inventario o a una tematización de problemas, lo cual, si bien no excluye la reflexión teórica, no se enfoca en ella. Quizá la problemática de la investigación educativa es la pregunta por el pensamiento y la crítica a las preguntas por la educación. Es decir, se trata de interrogar acerca de por qué es importante pensar qué es educación, qué implica reflexionar sobre la educación, qué conlleva indagar sobre la educación o para qué saber qué es educación. Por su parte, las problemáticas de la investigación educativa pueden llevarnos a interrogarnos por la elaboración de materiales didácticos, el diseño de actividades de aprendizaje, la planeación y gestión educativa o escolar, la intervención educativa, la medición o evaluación educativa, e incluso la pregunta por la pedagogía como disciplina, etc. En todo caso, la elaboración de material didáctico o el diseño de actividades de aprendizaje son una problemática de la investigación en tanto se les examina bajo las interrogantes que van más allá de la resolución de problemáticas específicas. Es decir, habría que preguntar por qué es importante saber qué es la didáctica o por qué es primordial conocer qué es el aprendizaje, o bien por qué es importante pensar qué es y qué función tiene la escuela, y para qué reflexionar sobre qué es y cómo surge la infancia.

Asimismo, es importante decir que, en todo quehacer de investigación educativa, debe distinguirse la pedagogía de la educación. En principio, la primera es un campo disciplinario, con un conjunto de métodos, conocimientos y procedimientos específicos, como cualquier otra disciplina, que permite dar cuenta de problemáticas educativas. Por su lado, la educación, además de ser un proceso constitutivo de nuestra especie humana, de la sociedad y de la cultura, puede ser vista como un campo de conocimiento u objeto de estudio multirreferencial y profundo sobre el cual se plantea la problemática de la investigación educativa. Desde luego, parte del quehacer de la pedagogía es plantear la problemática de la investigación educativa desde lo que se conoce como filosofía y teoría de la educación, que son dos lentes que, además de mirar la educación como un conjunto de problemáticas - de enseñanza, aprendizaje, conducta, medición, calificación, capacitación, competencias, niveles escolares, financiamiento, políticas públicas nacionales o internacionales, tecnologías, etc.-, también se interrogan sobre las problemáticas que van desde el propio logos - la lógica, la racionalidad, la sintaxis misma de la producción 
del pensamiento educativo-, hasta la creación y destrucción de culturas, de formas de vida y de sujetos, lo cual es constitutivo de la propia problemática de la investigación educativa. En este sentido, la problemática de la investigación educativa se interroga por ese proceso constitutivo de lo humano, de la sociedad y de la cultura, y por aquel campo de conocimiento y objeto de estudio multirreferencial; mientras que la problemática de la investigación pedagógica analiza los métodos, conocimientos y procedimientos específicos de la disciplina.

Así como es importante distinguir la pedagogía de la educación, es primordial diferenciar la investigación educativa de la investigación pedagógica. De manera general, se puede decir que la investigación pedagógica trata las indagaciones que se hacen sobre la educación desde parámetros propios de la pedagogía como disciplina - a partir de la didáctica, la psicología del aprendizaje, la planeación y gestión educativa, etc.- - A su vez, la investigación educativa explora la educación desde múltiples enfoques teóricometodológicos, de las humanidades y de las ciencias sociales. Por ello, podemos decir que toda investigación educativa es constitutivamente multidisciplinaria, ya que la pregunta por la educación, no solo lo referente a lo escolar, se hace desde diversos saberes y metodologías disciplinares ${ }^{1}$.

Si convenimos en que la pedagogía es una disciplina institucional y que la educación es un proceso o hecho sociocultural universal, podemos entender que el interés de la pedagogía en la investigación educativa reside fundamentalmente en aquellos aspectos que dirigen su atención al núcleo mismo de las prácticas y de las experiencias educativas, tales como los aspectos psicológicos del aprendizaje, la importancia de la didáctica, el desarrollo del currículum, la planeación y la gestión educativa, etc. Por esto, es frecuente que a los estudiantes de posgrado en educación no necesariamente se les exijan objetos de estudio pedagógicos, sino objetos de estudio educativos, que pueden ir desde la filosofía de la educación, pasando por la sociología y la economía de la educación, hasta el interés pedagógico por la educación. Por ello, podemos decir que la investigación educativa no es lo mismo que la investigación pedagógica.

Preguntarse por la investigación educativa en el marco de la pedagogía remite a la ubicación del carácter multidisciplinario de la primera en un ámbito específico, que es la disciplina pedagógica; mientras que interrogar por la investigación pedagógica en el marco de la investigación educativa implica ubicar el quehacer mismo de la investigación en didáctica, en la psicología del aprendizaje, en administración educativa, etc. —en un ámbito más amplio que es la investigación educativa-.

La educación como objeto de estudio no es propia ni exclusiva de la pedagogía. Las preguntas por la educación son tan antiguas como la humanidad misma. La pedagogía es un campo disciplinario surgido en el siglo XIX que se ha encargado del estudio y de la crítica de la educación. La educación es inconmensurable a todo planteamiento disciplinario. Así como el saber y la sabiduría desbordan la filosofía, la educación excede a la pedagogía y a cualquier disciplina o ciencia que intente dar cuenta de ella.

1 En este sentido, resulta interesante que el Consejo Mexicano de Investigación Educativa [COMIE] reconozca a la investigación educativa como una de sus áreas de conocimiento y no a la investigación pedagógica. De hecho, lo que el COMIE muestra, con los estados de conocimiento publicados cada década, es la investigación sobre la investigación educativa (López et al., 2013). 
Por lo anterior, este escrito se enfoca fundamentalmente en dos aspectos:

1. En la reflexión teórica y conceptual de la investigación educativa $-\mathrm{y}$ no en la identificación de problemas educativos específicos que pueden ser investigados-.

2. En la problemática de la investigación educativa $-\mathrm{y}$ no en la problemática de la investigación pedagógica-.

\section{Lo "multi" de la investigación educativa: entre humanidades y ciencias sociales}

Como se ha dicho, la problemática de la investigación educativa es un asunto multidisciplinario y multidimensional que puede ser abordado desde diferentes corrientes de pensamiento, donde se concibe a la educación como un valor universal inherente a lo humano, vinculado, sin duda, con las propias prácticas educativas - enseñanza, aprendizaje, transmisión, tradición, formación, etc.- - En este sentido, la problemática de la investigación educativa no remite solo al quehacer de la pedagogía, sino a otras disciplinas, a otras formas de pensamiento que han problematizado la educación, desde la mayéutica y la ironía practicadas por Sócrates, pasando por el estoicismo de Séneca, los imperativos categóricos de Kant, la voluntad de poder de Nietzsche, las formas elementales de la vida religiosa en Durkheim, la mitología comparada en Dumézil, los imaginarios sociales en Castoriadis, las relaciones de poder, saber y subjetivación en Foucault, la ética protestante y el espíritu del capitalismo de Weber, hasta el toltecayotl, analizado por León-Portilla, y el cuerpo humano como ideología en López Austin. En fin, todo ello plantea distintas aristas de la problemática de la investigación educativa.

Investigar la educación es un asunto no solo de la pedagogía, sino también de las humanidades y de las ciencias sociales, lo cual implica que los acercamientos teóricos y metodológicos a la educación sean múltiples, diversos e incluso contradictorios. Aunado a esto, la construcción de objetos de estudio en investigación educativa no se limita a la descripción del referente empírico, sino a la posibilidad de mirar en lo común y corriente de la realidad lo extraordinario o lo oculto de lo evidente. En este sentido, quizá una de las deficiencias de la investigación educativa realizada por la pedagogía es considerar que el único trabajo válido de investigación pedagógica es la mera colecta de información a través de diversas técnicas como entrevistas, sondeo, grupo de enfoque, encuestas, entre otras, lo cual puede ser muy valioso para la investigación educativa si se analiza y se reflexiona crítica y teóricamente la información y los datos, para mirar el revés del guante o para aprender de nuevo a ver: parafraseando a Proust, para considerar que el verdadero viaje de descubrimiento no consiste en buscar nuevos paisajes, sino en tener nuevos ojos.

Se coincide con Pontón (2011) en que

la construcción de nuevos conocimientos en los distintos campos disciplinarios se orienta hacia la transformación o construcción de problemáticas particulares relacionadas con el objeto de estudio de cada uno de ellos. Y esto se da a través de un proceso de articulación de las teorías, las categorías, los conceptos y los diversos procedimientos metodológicos que tienen como finalidad dar cuenta de las múltiples relaciones que se establecen en torno a un objeto de estudio. (p. 136)

La educación, vista como un objeto de estudio, puede ser problematizada por diversos 
campos disciplinarios como la sociología, la pedagogía, la economía, la psicología, la semiología, la antropología, la historia, la filosofía, la filología, entre otras. Por eso, hablar de investigación educativa conlleva no solo un enfoque multidisciplinario, sino también uno multirreferencial, en tanto métodos, conceptos, categorías e índices. La investigación educativa evidencia la articulación de diferentes enfoques disciplinarios que se interrogan por la educación a partir de sus propios referentes teóricos y perspectivas metodológicas.

La pedagogía es un campo de conocimiento que se acerca a la educación con miradas teóricas que comparte con otras disciplinas. Al mismo tiempo, la pedagogía tiene una dimensión práctica y técnica muy propia y clara. De tal manera, la problemática de la investigación educativa permite a la pedagogía teorizar la práctica y practicar la teoría.

Ubicar la investigación educativa dentro de las investigaciones en humanidades y ciencias sociales implica aceptar que la educación, además de ser un objeto de estudio que puede construirse a partir de diversas posturas teórico-metodológicas, propias de las ciencias sociales, también es, quizá, el elemento humanizante —o de producción de subjetividadesmás radical. Por ello, se acepta que los fenómenos, hechos o realidades educativas, culturales y sociales son inconmensurables en cuanto a conceptos, teorías y marcos metodológicos. No hay teoría, concepto, categoría o metodología que agote la realidad humana y el hecho educativo, social o cultural.

Sin embargo, hay aproximaciones metodológicas, propias de las ciencias sociales, desde las cuales puede investigarse la educación, por ejemplo: la inductiva, la deductiva, la cualitativa, la cuantitativa, la mixta, la experimental, la estadística, la etnometodológica... Desde las humanidades se puede investigar la educación a partir de enfoques hermenéuticos - Dilthey, Ricoeur y Gadamer-, del materialismo dialéctico -Marx-, pragmáticos - Peirce y Dewey-, genealógicos — Nietzsche y Foucault-, fenomenológicos - Husserl-, mitología comparada —Dumézil—, entre otros. Por ejemplo:

(a) La etnometodología pretende hacer observables e informables los fenómenos. Es decir, los sucesos de la vida común y corriente al organizarse, conceptuarse, categorizarse e indizarse, describen, narran y se hacen visibles como fenómenos. Desde luego,

ni el sentido reconocible, ni los hechos, ni el carácter metódico, ni la impersonalidad, ni la objetividad de las explicaciones que se dan son independientes de las ocasiones socialmente organizadas de su uso. En cambio, sus características racionales consisten en lo que los miembros hagan con, y hagan de, los relatos en las ocasiones concretas y socialmente organizadas de sus usos. Las explicaciones que dan los miembros están reflexiva y esencialmente vinculadas, en sus características racionales, a las ocasiones socialmente organizadas de sus usos, precisamente porque esas explicaciones son rasgos de las ocasiones socialmente organizadas de esos usos. (Garfinkel, 2006, p. 441)

La etnometodología aporta a la problemática de la investigación educativa, ya que provoca la reflexión teórica y conceptual sobre la realidad cotidiana. Si aceptamos que la problemática de la investigación educativa es la pregunta por el pensamiento y la crítica a las preguntas por la educación, desde la etnometodología puede plantearse la pregunta: ¿cuál es el sentido reconocible de la educación en tanto rasgos de las ocasiones socialmente organizadas para su uso? Es claro que las cualidades de esta pregunta no están encaminadas a hacer un inventario o una tematización de problemas específicos. En contraste, buscan 
indagar en los fondos de aquello que hemos llamado educación. Es una muestra de lo que la investigación educativa puede aprovechar de las aportaciones teóricas y metodológicas de las ciencias sociales.

Aun con esta propuesta metodológica, se busca no perder el sentido autocrítico respecto de la producción de conceptos, categorías e índices que someten y dan cierto sentido a una realidad racionalmente calculada. No olvidemos que

de un lado, la investigación de los fenómenos que se nos presentan como regulares, lo social, que en apariencia permanece idéntico a sí mismo, como eternamente instituido y de otro, lo irregular, el azar, lo cultural que irrumpe modificando, instituyendo para bien y para mal lo social. Las teorías intentan con-figurar la realidad. (Jiménez, 2012, p. 24)

(b) Por su parte, cuando se pretende abordar la educación desde la fenomenología de Husserl, se busca la suspensión - o epojé- como puesta entre paréntesis del mundo naturalmente educativo para que aparezca dicho mundo con una nueva significación. En otras palabras, a través del método fenomenológico, se pretende reconsiderar o resignificar los contenidos de la conciencia que se tiene sobre la educación. Al recordar a Husserl (1998), a través de la reflexión aprehendemos las vivencias subjetivas que llegan a ser para nosotros conscientes en tanto se nos aparecen, de ahí que todas estas vivencias conscientes se llamen también fenómenos. La fenomenología permite ver la otredad de la realidad. Tiene un modo de ver por medio del cual se ve todo lo suspendido. En palabras de Husserl (1998):

La epojé universal respecto del mundo que llega a ser consciente (su 'puesta entre paréntesis') desconecta del campo fenomenológico el mundo que para el sujeto en cuestión pura y simplemente existe, pero en su lugar se presenta el mundo así y asá consciente 'como tal', el 'mundo entre paréntesis'; o lo que es lo mismo en lugar del mundo o en lugar de algo mundano singular puro y simple, se presenta el respectivo sentido de consciencia en sus diferentes modos. (p. 43)

Con el método fenomenológico, la investigación educativa tiene un modo de ver a la educación, que permite la develación de las vivencias subjetivas al ponerlas entre paréntesis. Así, la fenomenología posibilita que la investigación educativa pueda partir de la pregunta: ¿cómo pasar de lo mundano de la educación a la consciencia de dicho fenómeno? El giro fenomenológico de la educación implica voltear la mirada de lo natural de este fenómeno a la conciencia de la experiencia que es la educación. Preguntar por "la realidad otra" de la educación conlleva un planteamiento propio de la problemática de la investigación educativa.

(c) Por otro lado, la genealogía es, como observó Deleuze (1998), "el valor del origen y el origen del valor"(p. 9). Es decir, desde la genealogía, como planteamiento teórico y metodológico para pensar la educación, habría que preguntar cuál es el valor del origen y el origen del valor de la educación. No se trata de preguntarnos por el inicio de la educación. Antes bien, desde la genealogía se interroga por los momentos en que las sociedades y las culturas empiezan a valorar algo distinto a lo hasta entonces valorado, lo cual impulsa un giro en la propia historia. De acuerdo con Nietzsche (1997),

hay que poner en entredicho el valor mismo de los valores, [el valor mismo de la educación] y para esto se necesita tener conocimiento de las condiciones y circunstancias de que aquellos surgieron, en las que se desarrollaron y modificaron. (p. 23) 
El método genealógico sirve a la investigación educativa, pues le permite entrar en las profundidades del ser del hombre como ser histórico. Es un regreso al origen de la educación en cuanto fondo y fundamento, un retorno que busca en aquellos fondos, los quiebres o desvíos que tuvo la educación. Las preguntas genealógicas que pueden plantearse desde la investigación educativa son: ¿en qué condiciones de vida se inventó el ser humano aquello que nombró como la educación? ¿Qué valor tienen las palabras y prácticas llamadas educativas? ¿En qué circunstancias históricas ha mutado la educación? Estos interrogantes no se dirigen a resolver un problema específico de la escuela o del aprendizaje, sino que se encaminan a la problemática de la investigación educativa y son pregunta por el pensamiento y la crítica a la educación.

(d) Por otra parte, Gadamer (2007) siguiendo a Heidegger, sostiene que

el círculo [hermenéutico] no debe ser degradado a círculo vicioso, ni siquiera a uno permisible. En él yace una posibilidad positiva del conocimiento más originario, que por supuesto solo se comprende realmente, cuando la interpretación ha comprendido que su tarea primera, última y constante consiste en no dejarse imponer nunca por ocurrencias propias o por conceptos populares ni la posición ni la previsión ni la anticipación, sino en asegurar la elaboración del tema científico desde la cosa misma. (p. 332)

Además, "toda interpretación [...] tiene que protegerse contra la arbitrariedad de las ocurrencias y contra la limitación de los hábitos imperceptibles del pensar y orientar su mirada a la "cosa misma»" (p. 333). La hermenéutica sirve a la investigación educativa en tanto le permite comprender la educación, es decir, proyectar nuevos sentidos sin negar los sentidos que le anteceden y sobre los cuales, sin ser arbitrarios, puede abrir la univocidad del lenguaje o del propio vocabulario de la educación y "definen" a la educación misma. Lo que se pretende, desde la hermenéutica, es la posibilidad de una teoría comprensiva que interprete la educación desde lo histórico y que sea parte de las ciencias del espíritu. Desde esta perspectiva teórico-metodológica, cuando en investigación educativa se pregunta qué es comprender la educación, esto lleva a indagar sobre cuáles son esos nuevos sentidos sobre los cuales, sin ser arbitrarios, puede abrir la univocidad del lenguaje o del propio vocabulario que nombran y "definen" la educación

Con los ejemplos anteriores, se observa que la problemática de la investigación educativa puede ser abordada desde diversas posturas epistemológicas propias de las humanidades y las ciencias sociales. Con ello se identifican dos aspectos:

1. Aunque se reconoce que dichas alternativas metodológicas corresponden a tradiciones teóricas y epistemológicas diferentes, es evidente que permiten pensar la investigación educativa y su problemática, desde posturas distintas a las comúnmente probadas.

2. La metodología es constitutiva del marco teórico, ya que, por ejemplo, no es lo mismo investigar la educación desde la genealogía que desde la etnometodología. Si bien toda fenomenología es hermenéutica, no toda hermenéutica es fenomenológica.

La problemática de la investigación educativa lleva a la educación a interrogar sobre la crítica y el pensamiento, lo cual implica preguntar cuáles son los sentidos reconocibles de la educación, cómo pasar de lo mundano de la educación a la consciencia de dicho fenómeno, cuál es el valor del origen y el origen del valor de la educación y cuáles son los sentidos 
no arbitrarios que pueden abrir la univocidad del lenguaje que nombra la educación. Estas preguntas solo pueden ser abordadas si se acepta el carácter multidisciplinario de la investigación educativa. La investigación educativa, en su sentido más amplio y profundo, no solo la realizada por la pedagogía, es una práctica, una acción, que se nutre de los enfoques y métodos propios, así como de los de las ciencias sociales y de las humanidades. La problemática de la investigación educativa implica atender el carácter multidimensional y multirreferencial de sus teorías y metodologías. Se puede decir que en una investigación educativa la teoría es constitutiva de la metodología. No puede definirse un objeto de estudio si la metodología elude su fundamento teórico.

Referirse a la educación como hecho social y cultural es atender una realidad compleja que está constituida por una serie de prácticas, procesos, contextos, instituciones, tradiciones, sujetos, referentes históricos, factores políticos y económicos, elementos geográficos y demográficos, incluso condiciones ambientales, cambios científicos y tecnológicos, entre otros, que en conjunto hacen posible y dan sentido a eso que se llama educación, formación humana, incluso cultura y procesos de subjetivación. Esto evidencia el carácter multidimensional y multirreferencial de la educación en tanto práctica y objeto de estudio.

Vista de esta manera, la educación solo puede ser estudiada a partir de referentes diversos, múltiples e incluso contradictorios, lo que hace de la investigación educativa algo "naturalmente" heterogéneo, plural y paradójico. La educación, como cualquier hecho sociocultural, nos coloca frente a una realidad que va más allá de los contenidos, parcelas de realidad o límites disciplinarios. Las preguntas acerca de cómo se forma el ser humano, cómo el ser humano se hace humano, cuáles y cómo son las prácticas que constituyen sujetos y qué hemos hecho que nos hace hacer lo que hacemos no son exclusivas del campo pedagógico, sino que atraviesan el terreno de la sociología, la política, la economía, la ética, la filosofía, la antropología e incluso el de la medicina, la psicología, la biología, la geografía, entre otras. Por ello, cuando se trata de investigar la educación, se habla no solo de investigación pedagógica, sino de la investigación educativa, la cual es constitutivamente multidisciplinar. Así, se acepta que la educación puede ser un proyecto sociocultural, al mismo tiempo que es un objeto de estudio multidimensional y multidisciplinario. Desde esta condición múltiple, es urgente estudiar la educación.

El prefijo multi-2 no solo se refiere a la abundancia y a la diversidad, en este caso, de las

2 Se sabe que en la actualidad se habla también de interdisciplina, transdisciplina, pluridisciplina y polidisciplina. Aunque los autores en muchos casos ofrecen definiciones diferenciadas entre estas nociones, en realidad son matices del mismo hecho que refleja la necesidad de una concepción más problemática y holística de la realidad en objetos de estudio más complejos. En un primer acercamiento a los matices que diferencian e integran las disciplinas para el estudio de la realidad, se encuentra que:

- Multidisciplina es la yuxtaposición de disciplinas distintas, donde no se especifican las relaciones entre ellas. Es solo un mero agrupamiento (Apostel et al., 1979).

- Pluridisciplina es la yuxtaposición de disciplinas distintas, cercanas a un mismo campo de conocimiento, que cooperan entre sí a partir de sus propios dominios (Palmade, 1979).

- Crossdisciplina - o disciplina cruzada - es la interacción entre dos o más disciplinas que comparte información a partir de una postura de fuerzas o de la determinación de un nivel jerárquico. Se trata de que las disciplinas crucen información en la realización de objetos de estudio. Por ejemplo, la historia de la ciencia recurre a métodos propios de las humanidades y las ciencias sociales (Torres, 1996). Y viceversa, las humanidades y las ciencias sociales toman conceptos de la ciencia, en particular, de la física, de la matemática y de la biología.

- Interdisciplina es la interacción entre dos o más disciplinas, que mantienen diversos canales de comunicación, donde incluso hay la integración de conceptos, términos, métodos, procesos, organización de datos (Apostel et al.,1979).

- Codisciplina - o transdisciplina - es el "conjunto de las concepciones que permiten unificar el conocimiento de las diferentes disciplinas manteniendo la originalidad de cada una de ellas. La concepción de la transdisciplinariedad desarrollada por Jantsch parece corresponderle" (Palmade, 1979, p. 220).

En realidad, aunque hay ciertos acuerdos entre los límites respecto a estas y otras agrupaciones de las parcelas de conocimiento, 
disciplinas. Antes bien, alude a las intersecciones, desvíos, irrupciones y articulaciones de ellas. Las disciplinas constituyen el vínculo entre ciencia y enseñanza, porque es a través de ellas que se institucionaliza el conocimiento, la verdad y los métodos. Son las disciplinas las que organizan los contenidos que deben ser enseñados. "No es exagerado pensar que en el origen [...] de la Universidad se encuentra la especialización [o disciplinarización] del conocimiento" (Pedroza, 2006, p. 71). O, en palabras de Apostel et al. (1979), "lo que concierne a la disciplina concierne, pues, a toda la estructura social de la Universidad" (p. XIII). Sin duda el trabajo de Le Goff (1990) respecto al papel de los intelectuales en la Edad Media ofrece fundamentos sólidos de la inevitable relación entre disciplina y universidad. Por ello, el carácter multidisciplinario de la investigación educativa - particularmente en lo referente al vínculo entre humanidades y ciencias sociales - toca la columna vertebral de la estructura académica de la universidad. Es decir, de lo que se trata es de colocar la mirada en torno al quehacer científico y didáctico de la universidad.

El carácter "multi" de la disciplina, además de ser un vínculo entre ciencia y enseñanza, también articula a la universidad con la empresa y el gobierno: estos demandan la producción y reproducción de conocimientos potencialmente útiles. Como se ha dicho en otros trabajos (Jiménez \& Valle, 2018), es interesante encontrar el vínculo de la universidad con la producción económica porque, en la actualidad, la crítica más común al trabajo de los universitarios, a sus concepciones, es la relación que se establece con la vida productiva, como si esto representara una degradación o desviación de los fines universitarios, como si la ciencia, la tecnología, la producción económica, la administración y el control de la sociedad y de los individuos no tuvieran que ver con el trabajo de la universidad. Lo "multi" de las disciplinas que hay en la universidad, particularmente cuando de investigación educativa se trata, puede permitir que se identifiquen y se analicen intersecciones, desvíos, irrupciones y articulaciones de los problemas propios de las actividades empresariales y gubernamentales. Lo que se pretende resaltar es que el carácter multidisciplinario de la problemática de la investigación educativa evidencia y fortalece el vínculo entre universidad y producción económica. ¿Qué sería de la filosofía y de las prácticas de los filósofos si no vincularan sus reflexiones con los problemas de la realidad actual, tales como tecnología -ingenierías-, migraciones y violencia - antropología, sociología, política, medicina, historia-, medio ambiente — geología, biología, geografía—, salud —medicina—, todos ellos enmarcados en la economía del mercado?

La relación de la universidad con el gobierno y la empresa es de antaño, ya Kant lo decía:

Entre las Facultades superiores [medicina, derecho y teología] solo se cuentan aquellas a cuyo respecto el gobierno mismo tiene interés en saber si sus enseñanzas son de tal o cual naturaleza, o si deben ser expuestas públicamente: en cambio aquella que solo está para velar por el interés de la ciencia se llama facultad inferior [filosofía], porque tiene libertad para disponer a su agrado de sus proposiciones. (Kant, 2004, pp. 21-22)

La división de las disciplinas en facultades desaparece cuando se trata de enfrentar no solo la comprensión de la realidad, sino también las demandas de aplicación y utilidad del conocimiento. La investigación educativa trata de aproximar a los estudiantes, profesores

unas de otras pueden ser consideradas como variaciones. Independientemente del prefijo puesto al sustantivo, lo que interesa es la integración del conocimiento, a través de las disciplinas, que trata de reflexionar en torno a las fronteras disciplinarias al mismo tiempo que piensa más allá de ellas. 
e investigadores de pedagogía - en particular, de ciencias sociales y humanidades- a la reflexión sobre la importancia de analizar, comprender o explicar problemas reales, demandados o no por empresas y gobiernos, desde una perspectiva integral y múltiple -dimensional, referencial y disciplinaria-.

Con base en lo anterior, el carácter multidisciplinario de la investigación educativa evidencia cruces que hay entre humanidades y ciencias sociales, y obliga a contar con posicionamientos teórico-metodológicos sólidos que permitan pensar la educación más allá de parcelas disciplinarias.

\section{Disciplinarización del saber en la investigación educativa}

Lo "multi" de la problemática de la investigación educativa no está exento de lo que Foucault ha llamado "disciplinarización del saber". La investigación educativa no deja de estar enmarcada en la investigación moderna y esta siempre se ha asociado a principios y prácticas científicas, con métodos experimentales que además de pretender objetividad y rigurosidad por aquello que se pregunta, también tienen, sobre todo, pretensiones de verdad, -la cual, en todo caso, según Foucault, se encarna en las prácticas de disciplinarización de los saberes-. La idea de una pedagogía científica basada en la experimentación que germina con el racionalismo del siglo XVIII más bien está ligada a los saberes educativos que entran en combate con otros saberes. En ese siglo, no se trataba tanto de la lucha entre luz y sombra, día y noche, conocimiento e ignorancia. Lo que surge es la guerra entre saberes, de unos contra otros. La pedagogía y el saber sobre la educación no escapan a la disciplinarización de los saberes, característica del siglo XVIII que ocurre cuando

un inmenso y múltiple combate [...] de los saberes que se oponen entre sí por su morfología distintiva, por sus poseedores que son mutuamente enemigos y por sus efectos de poder intrínsecos. [...] [lo que pasó en ese siglo fue] la existencia plural, polimorfa, múltiple, dispersa, de saberes diferentes, que existían con sus diferencias según las regiones geográficas, según la magnitud de las empresas, los talleres [...] según las categorías sociales, la educación, la riqueza de quienes los poseían [...] Por lo tanto, saber múltiple, saber secreto, saber que funcionaba como riqueza y garantía de independencia: el saber tecnológico funcionaba en esa fragmentación. (Foucault, 2014, pp. 167-168)

Los saberes tecnológicos se pueden entender como tecné en tanto: formas de hacer, prácticas, ejercicios, operaciones que sirven para producir o fabricar algo ${ }^{3}$. Así, lo que va a funcionar en medio de la multiplicidad y disciplinarización del saber es el saber tecnológico. El saber disciplinario en torno a la educación nació en y de este combate entre saberes polimorfos, múltiples, dispersos y secretos, que operaban como fuente de riqueza. La educación era un saber tecnológico que estaba en lucha con otros saberes y la pedagogía fue la ciencia que disciplinaría dichos saberes. La pedagogía disciplina la enseñanza y el aprendizaje, como saberes sobre la educación del ser y cuerpos humanos. Además,

a medida que se desarrollaron tanto las fuerzas de producción como las demandas económicas,

3 Estos saberes tecnológicos están muy cercanos a lo que Heidegger (1994) considera de la técnica como destino, aquella de la que el hombre, por su propia condición humana no puede escapar. Él señala que hay dos frases que responden a la pregunta sobre la técnica:

Una dice: la técnica es un medio para un fin. La otra dice: técnica es un hacer del hombre. Ambas determinaciones de la técnica se copertenecen. Pues poner fines, que utilizan y disponen medios para ellos, es un hacer del hombre. (p. 114)

La técnica implica una cara instrumental y otra antropológica. 
el precio de esos saberes aumentó, y la lucha de unos contra otros, las delimitaciones de la independencia y las exigencias del secreto se hicieron más fuertes y, en cierto modo, más tensas. (Foucault, 2014, p. 168)

Los saberes disciplinarios de la educación están, desde entonces, vinculados con las demandas económicas, con el establecimiento de precios, con el juego del mercado, con el liberalismo y ahora con el neoliberalismo4.

Sin duda, en el siglo XVIII, la disciplina encargada del saber de la educación hace referencia tanto a su naturaleza teórico-filosófica —ética y política fundamentalmentecomo a su quehacer práctico - en el ámbito escolar, principalmente-. Lo anterior está inmerso no tanto en un progreso ilustrado, de la luz sobre la oscuridad, del conocimiento sobre la ignorancia, sino que las raíces del saber disciplinario sobre la educación están en las disputas de los saberes tecnológicos caracterizados por su multiplicidad, independencia, heterogeneidad y secrecía. En otras palabras, como se ha dicho, la pedagogía, como ciencia de la educación, teoriza las prácticas y practica las teorías en las encrucijadas de los ejercicios y operaciones que sirven para producir lo humano, la formación humana, la cultura: la educación en su sentido más amplio y profundo.

En este proceso de disciplinarización, surge "la ciencia" - así en singular-. Las humanidades - en general - y la filosofía —en particular - pierden su lugar de saber fundamental. Se puede decir que la disciplinarización de los saberes evidencia el abandono de la exigencia de verdad y de saber, y se instaura el de la ciencia. Es decir, en la disciplinarización, la ciencia de la educación tiene prioridad sobre el saber y la verdad de la educación. En todo caso, el saber y la verdad están en función de la ciencia, sin dejar de reconocer que la educación - como hecho social y cultural, como forma humanizante más radical y como proceso de subjetivación - es inconmensurable en todo sentido, más allá de las disputas disciplinarias.

Con la disciplinarización de los saberes, hablar del carácter científico de la pedagogía o de la cualidad experimental de la investigación educativa implica reconocer la aplicación a lo social y a lo cultural de las metodologías y teorías de las ciencias naturales y las de las ciencias exactas. Cabe recordar que, como señala Valle (2015), la condición étima de la palabra pedagogía conlleva el estudio del niño o el estudio y conducción del niño, y que,

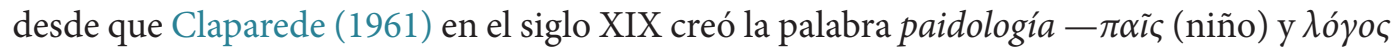
(estudio o ciencia) - para designar el nombre de una rama de la psicología, el hacer del investigador en educación no se circunscribe ni al gusto ni al estudio de la infancia. Aun sabiendo que el porvenir de la pedagogía científica fue a partir de la inauguración de la "Biblioteca de Pedagogía y Psicología" por Binet y Henri en el París de 1898, el hacer del

4 Aunque no es propósito de este texto reflexionar en torno a la relación entre investigación educativa, liberalismo y neoliberalismo, según Foucault (2016), el liberalismo, surgido en el siglo XVIII trata de una forma o arte de gobernar que parte de la fórmula laissez faire, laissez pase. Es un dejar hacer y dejar pasar del mercado que limita y regula las formas y los ámbitos de acción del Estado. El laissez faire es una consecuencia política de la economía de mercado. El liberalismo produce y consume libertad, sobre todo libertad de intercambio, es decir de mercado. Nada está por encima del mercado, ni siquiera el Estado; nada está por encima del colectivo, ni siquiera el individuo. Se trata de que el Estado no intervenga en las acciones del mercado y de que los intereses individuales no estén por encima de los intereses colectivos. Por su parte, el neoliberalismo trata de la entrada del Estado en el mercado: ya no se busca dejar hacer y dejar pasar del mercado que limita y regula las formas y los ámbitos de acción del Estado, como ocurre en el liberalismo. Ahora el Estado es parte de la economía política del mercado. En el neoliberalismo no solo se produce, se gestiona y se asegura la libertad, sino también la pluralidad de libertades que movilizan la competencia, el monopolio, la política social, el capital humano y la criminalidad. Será interesante revisar las peculiaridades de la disciplinarización del saber educativo en el marco del liberalismo y neoliberalismo. 
pedagogo y del investigador en educación no queda atado exclusivamente al positivismo experimental más estricto. Como se ha dicho, ese hacer está en el combate que es la disciplinarización de los saberes sobre la educación.

Cierto es que las preguntas por la observación y la experimentación en torno a la conducta humana sostienen el trabajo científico de lo que se convirtió en la pedagogía. "A medida que avanza el siglo XIX se siente cómo el progreso de las ciencias, causa y efecto a la vez de la revolución industrial, habrá también de afectar a la psicología y a la pedagogía" (Landsheere, 1996, p. 34), afección que no es otra cosa que el combate entre saberes técnicos y la disciplinarización de los saberes sobre educación. Se instala, por decirlo de alguna manera, una lucha de lo económico-político en torno a los saberes - en general- y, en particular, a los educativos.

Este reconocimiento de la aplicación a lo social y a lo cultural de las metodologías y de teorías de las ciencias naturales y la de las ciencias exactas es un momento teórico y metodológico que influyó en la manera de concebir la investigación educativa. Lo interesante aquí es que la problemática de la investigación educativa puede mantener dos aspectos constitutivos de su quehacer: primero, el combate propio de los saberes disciplinarios en torno a la educación - por ello, aún se habla de sociología de la educación, psicología de la educación, economía de la educación, filosofía de la educación, administración y gestión de la educación-; y, segundo, la tensión de las diferentes improntas positivistas del pensamiento, tales como el racionalismo, el empirismo y el nominalismo. Ejemplo de ello en un gran nivel es el propio Kant y pedagogos como Pestalozzi, Montessori y Decroly, por mencionar algunos.

Pestalozzi, el padre de la pedagogía contemporánea, a principios del siglo XIX, propuso la investigación empírica en la educación y planteó problemas de la llamada pedagogía experimental. En el mismo periodo, el propio Kant y su sucesor Herbart sostienen la necesidad de las escuelas experimentales. Por su parte, entre finales del siglo XIX y principios del XX, Montessori y Decroly fueron dos de los principales promotores de la llamada pedagogía científica, basada en la naturaleza del desarrollo del niño. Ambos, desde la medición y observación de niños anormales, propusieron, cada uno por su parte, un método científico. Además, están los trabajos de Durkheim y Dewey. El primero ofrece una perspectiva sociológica del conocimiento para analizar los hechos sociales, entre los que se encuentra la educación a la que le dedica parte de su vida y obra. Por su parte, Dewey presenta estudios sobre la conformación de la educación como proceso social.

Estos autores - por mencionar quizá a los más emblemáticos del siglo XVIII en adelanteson referentes clave para el análisis sobre la delimitación teórica - histórica y filosófica- de la pedagogía como campo disciplinario, el cual, desde la teoría práctica y práctica teórica, configura lo que puede llamarse la problemática de la investigación educativa. No podemos olvidar que el abordaje teórico-conceptual, filosófico, histórico y epistémico sobre la educación conforma la propia problemática de la investigación educativa. Es decir, mira a la educación como un problema de investigación multidisciplinario y multirreferencial.

Sumado a lo anterior, de acuerdo con Foucault (2014), el Estado, desde el siglo XVIII, interviene directa o indirectamente en las luchas entre saberes. Es decir, disciplina los saberes técnicos a través de cuatro procedimientos que mantienen una lógica fundamentalmente 
económica:

1. Eliminación o descalificación de los pequeños saberes inútiles e irreductibles, económicamente costosos.

2. Normalización de los saberes entre sí, lo que va a permitir ajustarlos unos con otros, establecer comunicaciones entre ellos, echar abajo las barreras del secreto y las delimitaciones geográficas y técnicas. Así los saberes y quienes los poseen se hacen intercambiables.

3. Clasificación jerárquica de los saberes que permite, en cierto modo, encajar unos con otros.

4. Centralización piramidal que permite el control de esos saberes que asegura la selección y posibilita la transmisión, desde abajo hacia arriba y viceversa. (p. 168)

Selección, normalización, jerarquización y centralización serán cuatro aspectos propios de la disciplinarización de los saberes. Desde entonces, todo saber sobre la educación, toda investigación educativa, fundamentalmente, tendrá una raíz en esta disciplinarización. En la lucha económico-política en torno a los saberes se encuentra el proyecto de la enciclopedia y el de la universidad moderna, que se encarga de seleccionar saberes, desaparecer el saber amateur e institucionalizar el conocimiento y las prácticas científicas. Basta ver cómo se presenta esta relación entre disciplinarización del saber y lucha económico-política en la institucionalización de la investigación educativa. El nacimiento y la extinción de instituciones de investigación educativa hacen evidente que los saberes disciplinarios no pueden eludir su vínculo con las demandas de la economía política del mercado.

\section{Reflexiones finales}

En suma, en este texto se trata una problemática —en singular-, lo cual remite a una indagación teórico-conceptual, y no problemáticas —en plural一, que pueden referirse a un inventario de problemas. En este sentidola problemática de la investigación educativa permite a la pedagogía teorizar la práctica y practicar la teoría. Se hace referencia a la investigación educativa como un campo multidisciplinario, y no a la investigación pedagógica, como un campo disciplinario específico. El prefijo multi- no solo indica la abundancia y diversidad de las disciplinas, ya que alude a las intersecciones, desvíos, irrupciones y articulaciones de ellas.

La problemática de la investigación educativa es un campo donde convergen preguntas, teorías, problematizaciones y metodologías diversas tanto de las humanidades como de las ciencias sociales, lo que implica que la pregunta por la educación no sea unívoca y cerrada. Más bien, conlleva la multirreferencialidad que siempre permite abrirse a nuevas preguntas y a la construcción de objetos de estudio.

La disciplina es el núcleo de cualquier agrupación de parcelas de conocimiento y es una bisagra entre ciencia y enseñanza, así como entre universidad y empresa-gobierno. La investigación moderna siempre se ha asociado a principios y prácticas científicas, con métodos experimentales que pretenden no solo objetividad y rigurosidad por aquello que se pregunta, sino, sobre todo, la verdad, que, en todo caso, según Foucault, se encarna en las prácticas de disciplinarización de los saberes. La idea de una pedagogía científica basada en 
la experimentación que germina con el racionalismo del siglo XVIII está ligada a los saberes educativos que entran en combate con otros saberes.

El saber disciplinario en torno a la educación nació en y del combate entre saberes polimorfos, múltiples, dispersos, secretos y que operan como fuente de riqueza. En tal sentido, la educación es un saber tecnológico que está en lucha con otros saberes y la pedagogía es la ciencia que disciplina dichos saberes. De igual modo, se puede decir que la didáctica disciplina la enseñanza, así como la psicología educativa disciplina el aprendizaje.

La educación solo puede ser estudiada a partir de referentes diversos, múltiples e incluso contradictorios, lo que hace de la investigación educativa algo "naturalmente" heterogéneo, plural y paradójico. La educación, como cualquier hecho sociocultural, nos coloca frente a una realidad que va más allá de los contenidos, parcelas de realidad o límites disciplinarios.

Recorrer la historia de la investigación educativa implica mirarla tanto desde su conformación teórico-conceptual como desde su constitución institucional. Respecto a la primera, vemos el nacimiento de la pedagogía como ciencia en el siglo de las luces con claras improntas positivistas, donde la razón, la verdad, la ciencia y el método marcan el ritmo de las investigaciones sobre la educación.

El hacer del investigador en educación debe reconocer el carácter crítico propio del pensamiento científico. Según Kant, en El conflicto de las facultades (2004), la Facultad de Filosofía sería aquella que

independientemente de las órdenes del gobierno [de la empresa o del mercado] en lo que se refiere a sus doctrinas, tenga libertad, si no de dar órdenes, al menos de juzgar a todos los que se interesan por la ciencia, es decir, por la verdad, y en que la razón tenga el derecho de hablar con franqueza (p. 23).

El ejercicio científico del investigador en educación es esta capacidad de juicio franco con pretensiones de razón y verdad. Lo que importa aquí no es "la Verdad", ni "la Razón", ni "la Ilustración" en sí, como productos, como resultados acabados y cerrados; lo que interesa es el ejercicio, las actividades, las prácticas, las aptitudes de los investigadores en educación que hacen de la educación un objeto de estudio.

Que la razón tenga derecho a hablar con franqueza, como dice Kant (2004), es el ejercicio mismo de las humanidades y de las ciencias sociales, por lo que la pedagogía y toda investigación sobre la educación no pueden eludir esta condición. Se trata de una aptitud crítica, que será "el arte de la inservidumbre voluntaria, de la indocilidad reflexiva" (Foucault, 2003, p. 11). Por cierto, el pensamiento de Foucault -filósofo, historiador, antropólogo- evidencia la problematización de las prácticas que producen sujetos. El pragmatismo - por llamar de alguna manera al método y a las investigaciones de Foucaultsirve a la investigación educativa en tanto nos permite interrogarnos por los procesos de subjetivación (Valle \& Jiménez, 2017).

El momento esencial de la aptitud crítica, que es el ejercicio de todo investigador y por tanto del investigador en educación, es el conocimiento del conocimiento, el saber del saber o la pregunta por la pregunta. Como dice Mier (2012), "la reflexión sobre la educación es la pregunta acerca de la pregunta [...] la pregunta constituye la educación, pero emerge de ella con la fuerza envolvente: es inherente y a la vez extrínseca a la pregunta por el sentido" (p. 
19). Esta capacidad de preguntar por las preguntas es el ejercicio de la filosofía y teoría de la educación, tan propio de la problemática de la investigación educativa. El interrogar por la interrogación, que es el ejercicio pedagógico por excelencia, no da respuestas cerradas, correctas o fijas. Si bien es cierto son preguntas que demandan sentidos o significados, son preguntas que descomponen, generan tensión, desarticulan, perturban los sentidos, no solo los del mundo, sino los de la propia forma de ser de los humanos. La problemática de la investigación educativa es un operar sobre fondo, una exploración radical, lo cual no es un operar mecánico; más bien, es un hacer contemplativo o teórico, fundamental en toda investigación. Investigar la educación conlleva poner en práctica el pensamiento y la crítica sobre la producción de subjetividades. El quehacer del investigador en educación, sin importar dónde y con quiénes, es una práctica reflexiva y un ejercicio del pensar.

El reto que plantea la problemática de la investigación educativa y su carácter multidisciplinario es mirar con ojos nuevos la riqueza y profundidad de la tradición, al asumir que no toda innovación es mejoría ni toda tradición es decadencia. Se trata de observar, con ojos de águila, lo extraordinario de lo ordinario.

\section{Referencias}

Apostel, L., Berger, G., Briggs, A., Michaud, G. (1979). Interdisciplinariedad. Problemas de la enseñanza y de la investigación en las universidades. ANUIES.

Claparede, E. (1961). Psicología del niño y pedagogía experimental. Continental.

Deleuze, G. (1998). Nietzsche y la filosofía. Anagrama.

Foucault, M. (2003). Sobre la ilustración. Tecnos.

Foucault, M. (2014). Defender la sociedad. FCE.

Foucault, M. (2016). Nacimiento de la biopolítica. FCE.

Gadamer, H. (2007). Verdad y Método I. Sígueme.

Garfinkel, H. (2006). Estudios en etnometodología. Anthropos.

Heidegger, M. (1994). La pregunta por la técnica. Ediciones del Serbal.

Husserl, E. (1998). Invitación a la fenomenología. Paidós.

Jiménez, M., \& Valle, A. (2018). Sujetos y poderes universitarios. Conflicto, porvenir y autoafirmación de la Universidad. En M. Jiménez, \& A. Valle, Sociología y pedagogía. Defender la universidad (pp. 11-46). UNAM

Jiménez, M. (2012). Investigación educativa. Huellas metodológicas. SADE.

Kant, I. (2004). El conflicto de las facultades. Losada.

Landsheere, G. (1996). La investigación educativa en el mundo. FCE.

Latapí, P. (2008). ¿Recuperar la esperanza? La investigación educativa entre pasado y futuro. Revista Mexicana de Investigación Educativa, 13(36), 283-297.

Le Goff, J. (1990). Los intelectuales en la Edad Media. Gedisa. 
López, M., Sañudo, L., \& Maggi, R. (Coords.) (2013). Investigaciones sobre la investigación educativa. 2002-2011. COMIE, ANUIES.

Mier, R. (2012). Diálogo pedagógico. Reconocimiento y creación de sentido. En A. Valle (Ed.), Alteridad entre creación y formación. Reflexiones en torno a la cultura y la educación (pp. 19-32). UNAM.

Nietzsche, F. (1997). Genealogía de la moral. Un escrito polémico. Alianza.

Palmade, G. (1979). Interdisciplinariedad e ideologías. Narcea.

Pedroza, R. (2006). La interdisciplinariedad en la universidad. Revista Tiempo de Educar, $7(13), 69-98$.

Pontón, C. (2011). Configuraciones conceptuales e históricas del campo pedagógico y educativo en México. UNAM.

Torres, J. (1996). Globalización e interdisciplinariedad: el currículum integrado. Morata.

Valle, A. (2015). Formas de hablar entre filosofía y educación. En C. Pontón, \& A. Valle (Coords.), Filosofía y educación: vínculos y desarticulaciones (pp. 19-39). UNAM.

Valle, A., \& Jiménez, M. (2017). Pragmatismo y educación. Aproximaciones a la noción de método en Foucault. En Ó. Pulido, M. Suárez, \& Ó. Espinel (Comps.), Pensar de otro modo. Herramientas filosóficas para investigar en educación (pp. 29-49). Editorial UPTC. 